\title{
A Normalized SVD based Image Watermarking with an Enhanced Robustness and Imperceptibility
}

\author{
R. Surya Prakasa Rao ${ }^{1}$, Prof. P. Rajesh Kumar ${ }^{2}$ \\ ${ }^{1}$ Dept., of ECE, AU College of Engineering, Visakhapatnam, India. \\ ${ }^{2}$ Dept., of ECE, AU College of Engineering, Visakhapatnam, India. \\ reddisuryaprakash2014@gmail.com,rajeshauce@gmail.com
}

\begin{abstract}
Digital Image watermarking is one of the most active and challenging subjects in the information hiding research because it is an efficient solution to protect the copyright of the digital media. This paper proposes a new robust watermarking approach based on the selection of singular features of host image into which the watermark has to embed. The host image initially subjected to a normalization process to find the invariant features and then the obtained invariant features are subjected to Integer Wavelet Transform (IWT) followed by Normalized Singular value Decomposition (NSVD). NSVD normalizes the singular values of invariant sub bands which can effectively resist the attacks. Genetic Algorithm (GA) is used to normalize the singular values. After, the obtained normalized singular values are modified by adding the singular values of watermark image. At the embedding phase, Particle Swarm Optimization (PSO) was used to optimize watermarking constant. Numerous experiments are conducted over the proposed approach to evaluate the performance. The obtained experimental results demonstrates that the proposed approach is superior compared to conventional approaches and is able to provide efficient resistance over Gaussian noise, salt \& pepper noise, median filtering, cropping, rotation, contrast enhancement, scaling and Histogram Equalization attacks.
\end{abstract}

Index Terms: Digital image watermarking, IWT, SVD, GA, PSO, PSNR, NC, SSIM.

\section{INTRODUCTION}

Digital Image Watermarking $[1,2]$ is the process of insertion of image watermark in media content and its extraction, ifrequired, for authentication or ownership verification ofmedia content. A digital image watermark is a piece of informationthat is hidden directly in media content, in such a way thatit is imperceptible to a human observer [3]. Different types of watermarkingmethods for digital contents have been developed thatare classified into different categories depending upon theuse and the requirement of information required for theextraction/detection of watermark. To check the authenticityof a digital content fragile watermarking is used while, forthe purpose of copyright protection, robust watermarking isutilized. This classification is application-dependent. Basedon the information required for the extraction/detectionprocess watermarking schemes can be classified into blind,semi-blind, and non-blind categories. Also, one more categorizationis possible depending upon the domain of embeddingof watermark: spatial and frequency. A detailed reviewof watermarking schemes can be found in $[4,5]$. In digital image watermarking, there will be a common problem rises at embedding phase. i.e., the optimization of embedding constant. This constant varies from image to image and also varies based on environments. Hence there is a need to optimize embedding constant such that the watermarked image must be robust and imperceptible to any type of environments. Generally, the artificial intelligence techniques such as Genetic algorithm (GA), Particle swarm optimization (PSO), Ant colony optimization (ACO) etc., techniques will be used for optimization purposes. In [6], a novel watermarking approach was proposed by combining the both GA and PSO to obtain an improved performance in watermarking criterion. However, [6] decomposes the image through the most popular Discrete wavelet transform (DWT) which is having main problem of information loss. Due to the down sampling process in DWT, the extracted watermark and also the host image can't recover entire information. Thus, the quality of the watermark will be reduced. In [7], a block based watermarking is proposed based on Discrete Wavelet Transform (DWT) and Singular Vale decomposition (DWT). Initially, the host image was divided into the blocks of size 8 X8 and then processed for DWT. From the obtained sub bands, the approximation band was subjected to SVD. Similarly all blocks are processed and the largest singular value is selected for embedding. However, it is observed that it is not robust for some types of attacks such as image rotation and image flipping. Asthe largest singular values from each block of the imagehave a different tolerance limit of modification to embedthe watermark, it results in some visible distortions in thewatermarked image with single scaling factor.In this paper, a new image watermarking approach is proposed by considering the both GA and PSO as an optimization techniques. This paper focuses towards the enhancement of robustness and also the imperceptibility. This approach incorporates the host image normalization and then processes for portioning into blocks to resist rotation and scaling attacks. Then IWT is applied on every block and the obtained approximation 
band is subjected to normalized SVD. Here the normalized SVD is an extended version of SVD which normalizes the singular values through a constant. Then the obtained normalized singular values are processed for embedding. In this paper, the optimization problem is solved through Particle Swarm Optimization (PSO) and Genetic Algorithm (GA). The SVD optimization is carried out through GA and the optimization of embedding constant is carried out through PSO. The proposed approach is applied on various images and the performance was evaluated for various types of attacks. The rest of the paper is organized as follows: Section II illustrates the details of related work. Section III provides a review about the preliminary concepts used in this paper. Section IV illustrates the details of conventional approach completely. The complete details of proposed approach is given in section V. Section VI illustrates performance evaluation details and finally section VII concludes the paper.

\section{RELATED WORK}

In a robust image watermarking scheme, a trade-offalways exists among the two conflicting objectives, imperceptibilityandrobustness. So, the main goal of a robust image watermarkingscheme is to produce the watermarked image withlow quality degradation and high robustness. Therefore, in order toimprove these objectives, researchers have proposed severalwatermarking schemes implemented in spatial as well as transformed domain that find a compromise between thesetwo objectives. The spatial domain watermarking techniquesdirectly embed the watermark into the host image by altering the pixel values [8-11]. These methods generally are lessrobust to image and signal processing attacks and requiredlow computational efforts, while frequency domain methodstransform the representation of spatial domain into thefrequency domain and then modify its frequency coefficientsto embed the watermark. There are many transform domainwatermarking techniques such as discrete cosine transforms(DCT) [12], discrete Fourier transforms (DFT) [13-14], discrete wavelet transforms (DWT) [15-17], and singularvalue decomposition (SVD) [2, 18-20]. These methods typicallyprovide higher image imperceptibility and are muchmore robust to image manipulations, but the computationalcost is higher than spatial domain watermarking methods. The performance of watermarking methods was furtherimproved by combining two or more transformations [21-33]. The singular value decomposition (SVD) is extensivelyused in image watermarking field in recent years due toits features. However, various researchers pointed out thefalse positive detection problem in most of the SVD-based algorithms [7, 34-35]. To counter this problem, numerousresearchers have proposed improved versions of SVD-basedimage watermarking schemes.A robust image watermarkingscheme based on SVD that embeds the entire watermark is given in [20]. There are two versions of this schemedepending on the implementation of SVD, to entire coverimage and block-wise. The imperceptibility of an imagewatermarking scheme using block based SVD proposed in [19] is improved by incorporating compensation operation.According to this scheme, the damage in the quality due toinsertion of the watermark in the left singular vector matrixis compensated bymodifying the right singular vectormatrix. The host image is segmented into non-overlapping blocksof size 4 $\times 4$; then the embedding blocks are selected atrandom. The watermark bits are embedded by modifyingthe coefficients in the first column of the left singular vectormatrix of the target blocks. The different regions within animage have different local features, so some visual modelssuch as human visual system (HVS) may be incorporatedinfinding the suitable embedding regions to improve robustnesswhile maintaining imperceptibility. Based on this concept, ablind SVD-based watermarking scheme is presented in [36].The host image is segmented into non-overlapping blocks ofsize $8 \times 8$; then the embedding blocks are selected based on the sum of visual and edge entropies. The watermarkbits are embedded by modifying the coefficients in the first column of the left singular vector matrix of the target blocks. The above mentioned SVD-based watermarking schemesembed the entire watermark within the cover image. It has improved the reliability of the watermarking but sacrificedthe transparency. Also, these schemes are applicable only for the black and white watermark. A watermarking scheme proposed in [32] is based on thefact that SVD subspace (left and right singular vectors)can preserve a significant amount of information aboutan image. Therefore, it embeds the principal component,multiplication of left singular vector matrix and the singularvalue matrix, of watermark into the host image instead ofsingular values of the watermark. On the same concept,Run et al. [33] introduced an image watermarking scheme embedding the principal component of the watermark infrequency domain (DCT and DWT domains, resp.). Also,an optimization technique is applied to get the optimalscaling factors for embedding. Though the features extracted are robust for so many attacks, the efficiency of watermarking approach also depends on the watermarking constant. There are so many approaches proposed in earlier towards the optimization of watermarking constant. PSO is an intelligentalgorithm that using the stochastic, populationbased computeralgorithm for problem solving. Zheng [37] applied thePSO to search the embedding location of the integer DCT coefficientsin a block to optimize the requirement of imperceptibilityand robustness in watermarking. Vahedi [38] utilized the PSO method to search for the optimal energy ofembedding watermark to balance the quality and robustness ofwatermarked image. In [39], a watermarking approach was proposed based on Genetic Algorithm (GA). In [23], GA was used for the selection of watermarking constant. Recently, particle swarm optimization (PSO) wasevolved into the watermarking system. Hai Tao [40] applied PSO for the optimization of scaling factors to improve the robustness of watermarking scheme. 3-level DWT is used for 
feature extraction and PSO for optimization. Though the PSO was used, there is a non-recoverable information loss due to the 3-level DWT. In [41], a novel watermarking approach was proposed by considering the PSO as an optimizer and Integer wavelet transform as a transform technique. Though the information loss was reduced in [41], there observed a reduced robustness in particular attacks like rotation, histogram equalization and cropping.

\section{A. Integer Wavelet Transform (IWT)}

\section{PRLEMINARIES}

The main problem with wavelet transform is its inability to reduce the loss of information in the original image. For example, if any one of the block of original image having integer pixel values and transformed through a floating point wavelet transform. If the transformed coefficients are changed during the embedding, then this wavelet transform will not provide any guarantee about the integer values of that particular block. The truncation of floating point values will result in loss of information, i.e., the original image cannot be reconstructed effectively. Furthermore, the conventional wavelet transform is, in practice, implemented as a floating-point transform followed by a truncation or rounding since it is impossible to represent transform coefficients in their full accuracy. To avoid this problem, an invertible integer-to-integer wavelet transform based on lifting [41] is used in the proposed scheme. It maps integers to integers and does not cause any loss of information through forward and inverse transforms. The main advantage with Lifting based wavelet transforms is fast and accuracy. They are easy to implement and also doesnot require any additional memory.

The forward transform of a typical lifting scheme usually consists of three steps: split, predictionand update. Consider a signal: $X=\{x(n), n \in Z\}$ with $x(n) \in R$. The implementation of the forwardtransform is illustrated as below:

(1) Split: The original signal $X$ is split into two subsets: even indexed samples $x_{e}$ and odd indexed sample $x_{o}$ by means of a sample operation:

$\left\{\begin{array}{c}x_{e}=x(2 n) \\ x_{o}=x(2 n+1)\end{array}\right.$

After the split operation is completed, the odd set and even set are obtained and the two sets areclosely correlated. That is, adjacent samples are much more correlated than those far from each other. Itis natural that one can build a good predictor for one set with other set.

(2) Prediction: Given the odd indexed samples $x_{o}$, a predictor $P$ for the even indexed samples $x_{e}$ can be designed:

$\widetilde{x_{o}}=P\left(x_{e}\right)$

The difference denoted as $d$ between the predicted results and the odd samples is considered as thedetail coefficients of the signal $x(n)$, and it is expressed as:

$d=x_{o}-\widetilde{x_{o}}=x_{o}-P\left(x_{e}\right)$

(3) Update: Knowing the even sample $e x$ and the detail coefficients $d$, the approximation coefficients $c$ arecalculated using the updating operator $U$ as:

$c=x_{e}+U(d)$

The inverse transform can immediately be derived from the forward transform by running the liftingscheme backwards. The block diagram of the lifting scheme is given in Figure 1.

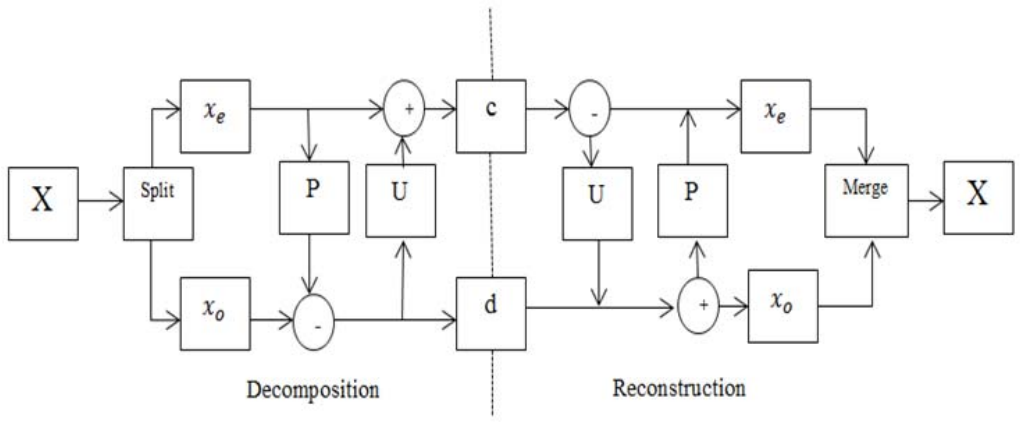

Figure.1 Lifting based decomposition and reconstruction

\section{B. Singular Value Decomposition (SVD)}

The singular valuedecomposition (SVD) [20] is a numerical analysis techniquebased on a theorem of linear algebra that decomposesa rectangular matrix into the product of three matrices:an orthogonal matrix $(U)$, a diagonal matrix $(S)$, and the transpose of an orthogonal matrix $(V)$. It may be consideredas a method of 
transforming correlated data set into uncorrelated one that better explains the various relationships among the original data. Due to the unique features and attractive properties such as stability with little disturbance, SVD has been used in many signal and image processing applications such as image watermarking, image hiding, image compression, and noise reduction. The digital image is also a kind of signal which can be viewed as a matrix. According to the theory, the SVD of a rectangular matrix Aof order $m \times n$ is represented mathematically as

$A=U S V^{T}$,

where $U U^{T}=I_{m}$ and $V V^{T}=I_{n}$; the columns of $U$ are orthonormal eigenvectors of $A A^{T}$, the columns of $V$ are orthonormal vectors of $A^{T} A$, and $S$ is a diagonal matrix containing the square roots of the eigenvalues from $U$ or $V$ in descending order. If $r(r \leq n)$ is the rank of the matrix $A$ then the elements of the diagonal matrix $S$ satisfy the relation (6) and the matrix $A$ can be written as (7):

$\lambda_{1} \geq \lambda_{2} \geq \cdots \geq \lambda_{r}>\lambda_{r+1}=\lambda_{r+2} \cdots=\lambda_{n}=0$,

$A=\sum_{k=1}^{r} \lambda_{k} u_{k} v_{k}^{T}$

Where $u_{k}$ and $\mathrm{V}_{k}$ are the $k_{\mathrm{th}}$ eigenvector of $U$ and $V$ and $\lambda_{k}$ isthe $k_{\mathrm{th}}$ singular value.

\section{Genetic Algorithm}

Genetic algorithm [39] is one of the most widely used artificial intelligent techniques belonging to the area of evolutionary computation. Usually, a simple GA is mainly composed of three operations: selection, genetic operation, and replacement. Initially, a population is randomly generated. The fitness function then uses objective values from objective function to evaluate the fitness of each chromosome. The fitter chromosome has the greater chance to survive during the evolution process. The objective function is problem specific; its objective value can represent the system performance index (e.g., an error). Next, a particular group of chromosomes is chosen from the population to be parents.

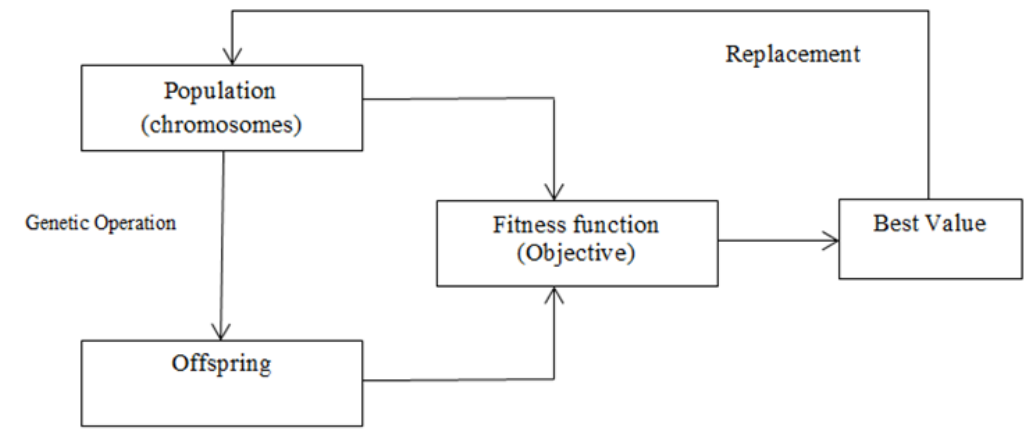

Fig.2. GA cycle

The offspring is then generated from these parents by using genetic operations, which normally are crossover and mutation. Similar to their parents, the fitness of the offspring is evaluated and used in replacement processes in order to replace the chromosomes in the current population by the selected offspring. The GA cycle is then repeated until a desired termination criterion is satisfied, for example, the maximum number of generations is reached or the objective value is below the threshold.

\section{Particle Swarm Optimization (PSO)}

PSO [40] is motivated from the social behavior of organism such as bird flocking or fish schooling. It attempts to mimic the natural process of group communication in a wide range of domains and can be used to solve many different problems. Like other evolutionary algorithms, PSO is also a population-based search algorithm and initializes with a population of randomly generated solutions called particles which fly through the search space by updating the generation. Each particle represents a candidate solution to the optimization problem, and has a velocity and a position. The position of a particle is affected by both the best position visited by it and the position of the best particle in its neighbourhood. The best particle in the population is denoted by global best (gbest),while the best position that has been visited by the current particle is denoted by local best(pbest). Each particle is updated using the following equations:

$v_{i}(n+1)=w_{i} v_{i}+c_{1} \operatorname{rand}_{1}\left(\right.$ pbest $\left.-x_{i}(n)\right)+c_{2} \operatorname{rand}_{2}\left(\right.$ gbest $\left.-x_{i}(n)\right)$

$x_{i}(n+1)=x_{i}(n)+v_{i}(n+1)(9)$

Where:

$x_{i}(n+1)$ and $x_{i}(n)$ represent the current and the previous positions of particle $\mathrm{i}$

$v_{i}(n+1)$ and $v_{i}(n)$ are the current and the previous velocity of the particle $i$. 
rand $_{1}$ and rand $_{2}$ are random numbers uniformly distributed within $[0,1]$.

$W$ is an inertia weight which controls the momentum of the particle.

In typical implementations of PSO algorithm, the value of $i w$ is decreased linearly from 1.0 to near 0 in each iteration. Commonly the inertia weight is set according to the following equation:

$w_{i}=w_{\max }-\frac{w_{\max }-w_{\min }}{\text { iter }_{\max }}$. iter

Where: iter $_{\max }$ is the maximum number of iterations, and iteris the current number of iterations. Each particle in PSO shares the information with its neighbors. The updating equations (4) and (5) combine both of the cognition component of each particle and the social component of all the particles in a group. Although the speed of convergence is very fast, many experiments have shown that once PSO traps into local optimum, it is difficult for PSO to jump out of the local optimum.

\section{IV.PROPOSED WATERMARKING SCHEME}

This section illustrates the complete details about the mathematical formulation of the proposed watermarking scheme and its components. The system developed for proposed watermarking approach is shown in figure.3.

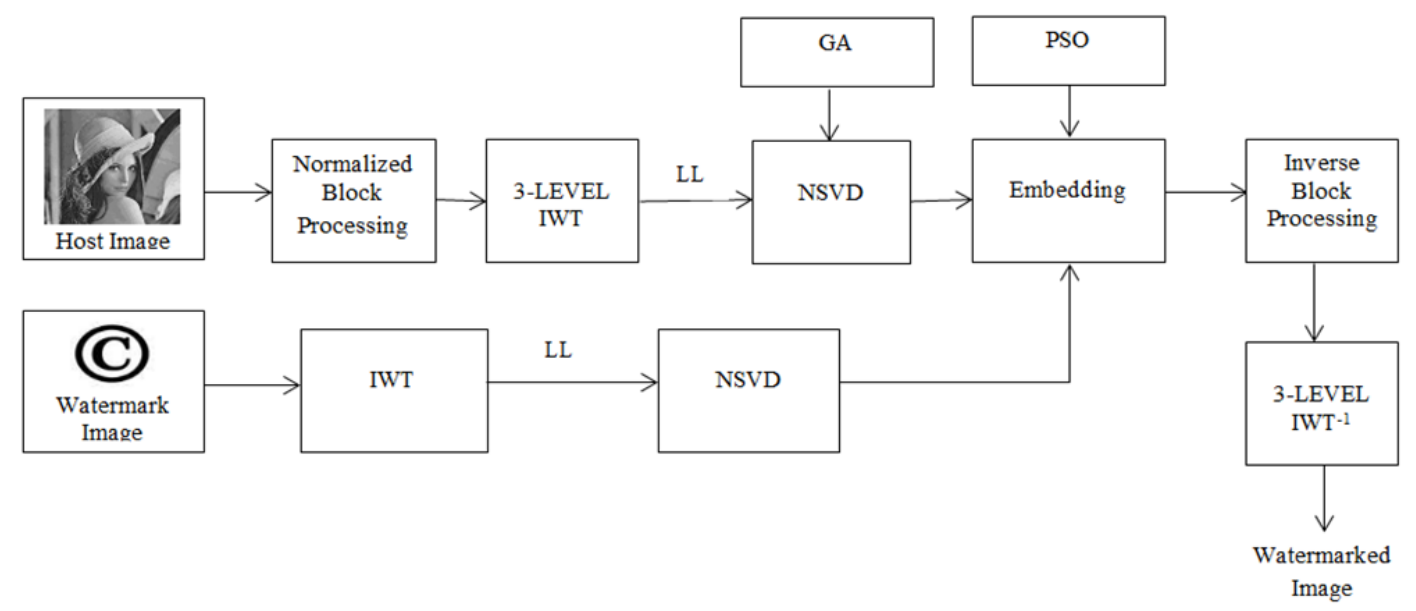

(a)

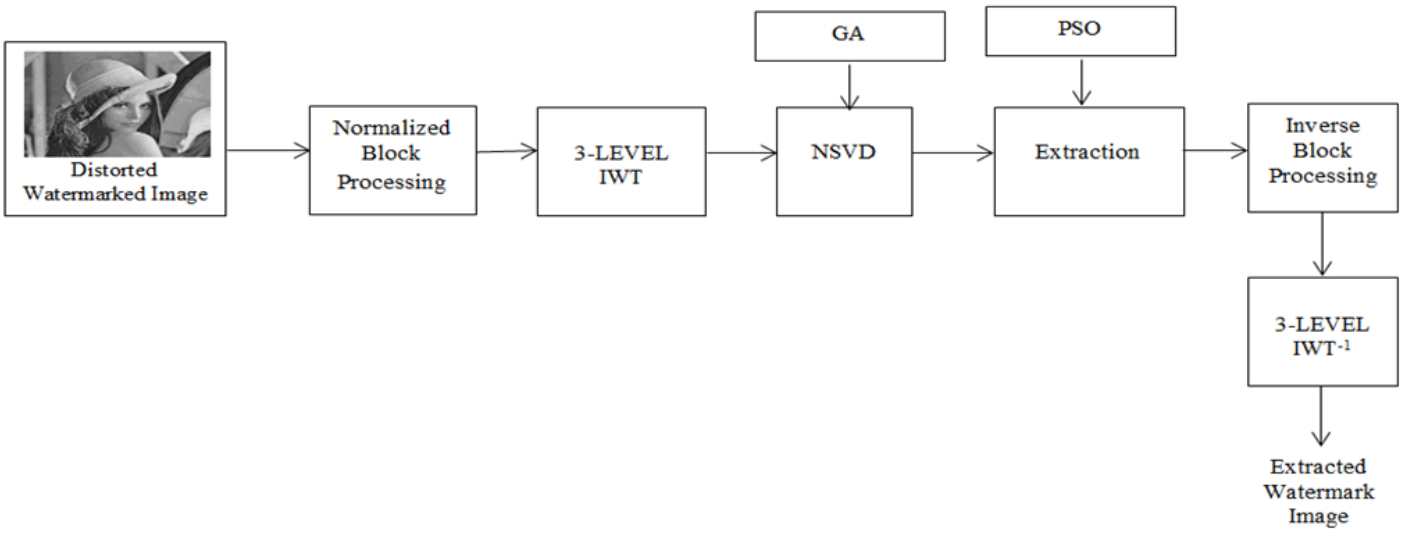

(b)

Figure.3. Proposed system for image watermarking (a) embedding phase (b) Extraction phase

\section{A. Normalized Block Processing}

The purpose of normalized block processing is to extract the invariant blocks of host image under rotation and flipping operations. The location of pixel values in a block may vary, but the elements of the blocks remain the same. To achieve it, pixels' locations of the image are redistributed and then some normalization procedures are performed. The block diagram of normalized block processing is shown in figure.4. Mathematically, it is formulated as follows.

Let $I$ be the host image of size $M \times N$ and Wbe the watermark image of size $K \times L$.

Step 1: Divide the host image I into four equal size blocks as B1, B2, B3 and B4. Evaluate mean for every block such that $m_{B 1}, m_{B 2}, m_{B 3}$ and $m_{B 4}$ are the means of $\mathrm{B} 1, \mathrm{~B} 2, \mathrm{~B} 3$ and $\mathrm{B} 4$ respectively. 
Step 2: Define a normalization mean matrix and sign matrix by summing and subtracting the means of all blocks, such as

$N_{i}^{m}=\left[\begin{array}{ll}N_{1}^{m} & N_{2}^{m} \\ N_{3}^{m} & N_{4}^{m}\end{array}\right]=\left[\begin{array}{ll}m_{B 1}+m_{B 2}+m_{B 3}+m_{B 4} & m_{B 1}+m_{B 2}-m_{B 3}-m_{B 4} \\ m_{B 1}-m_{B 2}+m_{B 3}-m_{B 4} & m_{B 1}-m_{B 2}-m_{B 3}+m_{B 4}\end{array}\right]$

$N_{i}^{S}=\left[\begin{array}{cc}N_{1}^{S} & N_{2}^{S} \\ N_{3}^{S} & N_{4}^{S}\end{array}\right], S=\operatorname{sign}=\{-1,+1\}$

Step 3: Obtain the normalized image by rearranging the original image according to the following rule,

$$
\left\{\begin{array}{cc}
N_{I}(2 k-1,2 l-1)=I(k, l) & 1 \leq k \leq M / 2,1 \leq l \leq N / 2 \\
N_{I}(2 k-1,2 l-N)=I(k, 3 N / 2-l+1) & 1 \leq k \leq M / 2, N / 2 \leq l \leq N \\
N_{I}(2 k-M, 2 l-1)=I(3 M / 2-k+1, l) & M / 2 \leq k \leq M, 1 \leq l \leq N / 2 \\
N_{I}(2 k-M, 2 l-N)=I(3 M / 2-k+1,3 N / 2-l+1) & M / 2 \leq k \leq M, N / 2 \leq l \leq N
\end{array}\right.
$$

Step 4: Then perform the 3-level IWT on the obtained Normalized image and multiply the sub band of normalized image with normalized sign matrix as shown in Equation.(12) and denote the obtained matrix as $S=\left(\begin{array}{ll}S_{1} & S_{2} \\ S_{3} & S_{4}\end{array}\right)$

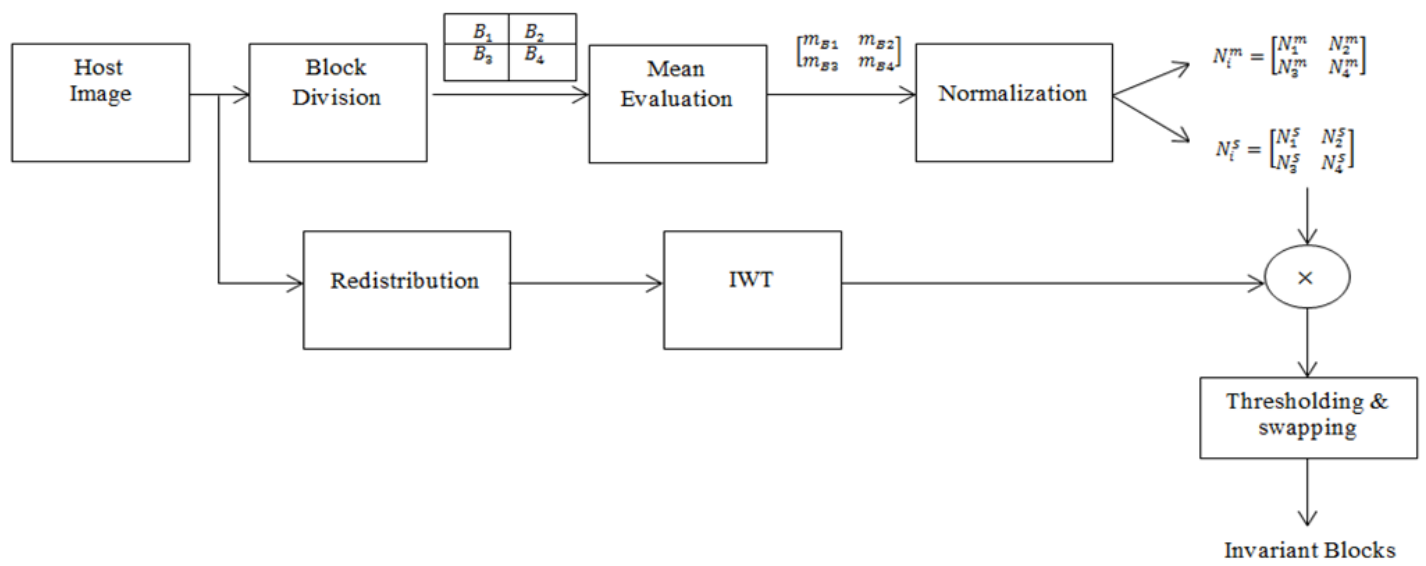

Fig.4. Normalized Block Processing \& IWT

Step 5: The obtained matrix $\mathrm{S}$ will be invariant if the condition $\left|N_{3}^{m}\right|<\left|N_{2}^{m}\right|$ is satisfied, otherwise, the swapping will be done and the final matrix was obtained by transposing the entire S matrix, i.e., $S=\left(\begin{array}{cc}S_{1}^{T} & S_{2}^{T} \\ S_{3}^{T} & S_{4}^{T}\end{array}\right)$.

The final matrix $\mathrm{S}$ is the invariant wavelet domain; it is invariant to rotation and image flipping. That is, once the original is flipped or rotated the same wavelet domain can be achieved by above normalized block processing.

\section{B. Normalized SVD (NSVD)}

From the SVD point of view it was noticed that every image matrix has the well-known SVD for any given single matrix $A$, the larger Singular Values (SVs) are very sensitive to variations in the image such as noise changes in the host image. Upon the occurrence of attack on the watermarked image, there may be effect on the pixel intensities. But the SVD are very sensitive to these variations. To alleviate the variations in image, a normalized SVD approach is proposed with mainly two ideas such as the weights of host image $u v^{T}$ should be deflated since they are every sensitive to the variations in the image itself and weights of base images $u v^{T}$ correspondingto relatively small $\lambda_{i}{ }^{\prime}$ sshould be inflated, since they may be less sensitive to the variations within the image.

This can be illustrated through the following concept. Let's consider the image $I$ is denoted as $I=$ $\left[i_{1}, i_{2}, \ldots, i_{r}\right]^{T}$, where $i_{i}^{T}$ is a $1 \times c$ row vector that represents the $i_{\text {th }}$ row of matrix $\mathrm{I}$, then

$V^{T} C_{\text {row }} V=S^{2}$

Where

$C_{\text {row }}=I^{T} I=\sum_{i=1}^{r} i_{i} i_{i}^{T}$ 
I.e., $v_{j}$ is the eigenvector of the covariance matrix $C_{\text {row }}$ corresponding to Eigen value $\lambda_{j}^{2}, j=1,2, \ldots, k$. Similarly, Let's consider the image $\mathrm{I}$ is denoted as $I=\left[i_{1}, i_{2}, \ldots, i_{c}\right]^{T}$, where $i_{i}^{T}$ is a $r \times 1$ row vector that represents the $\mathrm{i}_{\text {th }}$ Colom of matrix I, then

$U^{T} C_{\text {row }} U=S^{2}$

Where

$C_{\text {row }}=I^{T} I=\sum_{i=1}^{r} i_{i} i_{i}^{T}$

I.e., $u_{j}$ is the eigenvector of the covariance matrix $C_{\text {row }}$ corresponding to Eigen value $\lambda_{j}^{2}, j=1,2, \ldots, k$.

Hence, the $\lambda_{i}{ }^{\prime}$ sshould be inflated, since they may be less sensitive to the variations within the image, a new SVD formulation can be derived by modifying the standard SVD evaluation (shown in equation.(5)) as,

$A=U S^{\gamma} V^{T}$

Where $U, S$ and $V$ are the corresponding matrices in Eq. (1), and $\gamma$ is the normalizing constant. In order to achieve the requirements, the $\gamma$ needs to satisfy the following condition.

$0 \leq \gamma \leq 1$

C. Embedding Process

Step 1: Let $\mathrm{W}$ be the watermark image. Perform one level IWT on the Watermark image W

$$
W \rightarrow\left\{W_{L L}, W_{L H}, W_{H L}, W_{H H}\right\}
$$

Step 2: perform block processing on the LL band $W_{L L}$. Evaluate an optimal normalization constant $(\gamma)$ through the GA algorithm and then perform the normalized SVD (NSVD) on the obtained LL band of Watermark image $\mathrm{W}$

$$
W_{L L}(i, j) \rightarrow U_{W}(i, j) S_{W}^{\gamma}(i, j) V_{W}^{T}(i, j)
$$

Step 3: Obtain principal component by multiplying the components $U_{W}$ and $S_{W}^{\gamma}$.

$$
W_{U S}(i, j)=U_{W} S_{W}^{\gamma}
$$

Let $W_{U S}(i, j)$ be the principal component at pixel position $(i, j)$, where $i, j=1,2, \ldots, N$.

Step 4: Apply normalized block processing on the original host image to obtain normalized image.

Step 5: Apply three level IWT on the normalized image.

$$
N_{I} \rightarrow\left\{N_{I L L}, N_{I L H}, N_{I H L}, N_{I H H}\right\}
$$

Step 6: Evaluate an optimal normalization constant $(\gamma)$ through the GA algorithm and then perform the normalized SVD (NSVD) on the obtained LL band of Normalized Host image having invariant blocks to get the largest singular value $\lambda_{\max }$. Let $l l(i, j)$ be the invariant block at position $(i, j)$.

$$
l l(i, j) \rightarrow U_{l l} S_{l l}^{\gamma} V_{l l}^{T}
$$

Where $S=\operatorname{diag}\left(\lambda_{k}\right), k=1,2, \ldots, N$. The watermark principal component is embedded into the host image by modifying the largest singular value.

Step 7: Embed the watermark principal component into the largest singular value of host image for each image block $(i, j)$ using the following formula:

$$
\lambda_{\max }^{m}=\lambda_{\max }(i, j)+\alpha W_{U S}(i, j)
$$

Where $\lambda_{\max }^{m}$ is the modified singular value of image block $(i, j)$ and $\alpha$ is the watermarking constant can be obtained through the PSO.

Step 8: perform inverse SVD on the obtained singular values of every block $(i, j)$.

$$
l l^{m}(i, j) \leftarrow U_{l l}\left(S_{l l}^{\gamma}\right)^{m} V_{l l}^{T}
$$

Where $\left(S_{l l}^{\gamma}\right)^{m}$ is the modified singular matrix for the block at position $(i, j)$.

Step 9: reconstruct the complete LL band after getting individual blocks. Thus the reconstructed LL band will be a modified band of the normalized image, can be represented as $N_{I L L}^{m}$.

Step 10: perform Inverse IWT (IIWT) to reconstruct the normalized watermarked image $\left(N_{I}^{W}\right)$.

$$
N_{I}^{W} \leftarrow\left\{N_{I L L}^{m}, N_{I L H}, N_{I H L}, N_{I H H}\right\}
$$

Step 11: perform inverse redistribution and normalization to obtain the final watermarked image $I_{W}$.

\section{Extraction Process}

The extraction process is applied on the distorted watermarked image, $I_{W}^{*}$, to extract the watermark image and after the extraction the obtained watermark image is also distorted and can be represented as $W^{*}$. The following procedural steps were followed for extraction purpose. 
Step 1: Apply the normalized block processing on the distorted watermarked image $\left(I_{W}^{*}\right)$ to obtain the distorted normalized watermarked image $\left(N_{I_{W}}^{*}\right)$.

Step 2: Apply three level IWT on the distorted \&normalized watermarked image $\left(N_{I_{W}}^{*}\right)$.

$$
N_{I_{W}}^{*} \rightarrow\left\{N_{I_{W}^{L L}}^{*}, N_{I_{W}^{L H}}^{*}, N_{I_{W}^{H L}}^{*}, N_{I_{W}^{H H}}^{*}\right\}
$$

Step 3: Divide the approximate band into equal size non-overlapping blocks which can be denoted as $l l^{*}(i, j), i, j=1,2, \ldots, N$.

Step 4: Apply NSVD on every block $l l^{*}(i, j)$ to obtain the maximum singular value $\lambda_{\max }^{*}(i, j)$.

$$
l l^{*}(i, j) \rightarrow U_{l l}^{*}\left(S_{l l}^{*}\right)^{\gamma}\left(V_{l l}^{*}\right)^{T}
$$

Step 5: extract the distorted principal component $W_{U S}{ }^{*}(i, j)$ through the following formula as,

$$
W_{U S}{ }^{*}(i, j)=\frac{\left(\lambda_{\max }^{*}(i, j)-\lambda_{\max }(i, j)\right)}{\alpha}
$$

Step 6: Perform inverse NSVD by multiplying the obtained principal component $W_{U S}{ }^{*}(i, j)$ with $V_{W}^{T}$ to obtain a distorted block $W_{l l(i, j)}^{*}$

$$
W_{l l(i, j)}^{*} \leftarrow W_{U S}^{*}(i, j) V_{W}^{T}
$$

Step 7: Reconstruct the distorted LL band of watermark image by merging the all obtained distorted blocks.

$$
W_{L L}^{*} \leftarrow\left\{W_{l l(i, j)}^{*}\right\}
$$

Step 8: Perform Inverse IWT to obtain the final extracted watermark image $W^{*}$.

$$
W^{*} \leftarrow\left\{W_{L L}^{*}, W_{L H}, W_{H L}, W_{H H}\right\}
$$

Where $W^{*}$ is the extracted watermark.

This entire process repeats for entire population generated for optimization in GA and PSO.

\section{V.SIMULATION RESULTS}

In this section, the performance of proposed approach was analyzed under various experiments. For performance evaluation, three host mages and two watermark images are considered and shown in figure.5 and figure. 6 respectively. To investigate the robustness of proposed approach, the watermarked image was subjected to eight attacks such as: (1) Gaussian noise Attack (GNA) with noise variance as $0.001,0.01$ and $0.1,(2)$ salt \& pepper noise attack (SPA) with noise variance as 0.001, 0.01 and 0.1, (3) Median Filtering attack (MFA) with average window size of $3 X 3$ and 7X7, (4) Histogram Equalization attack (HEA), (5) Rotation attack (RA) with rotation of $30^{\circ}$ and $45^{\circ}$, (6) Contrast Enhancemnet attack (CEA) with contrats limit of 0.03 and0.05, (7) cropping attack (CA) and (8) Scalling Attack (SA) with Nearest Neighbor, bi-linear and bi-cubic.

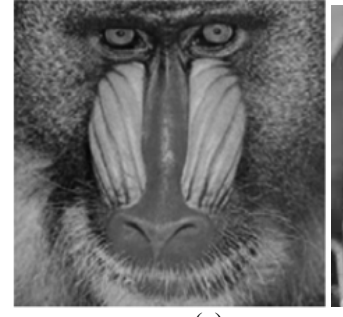

(a)

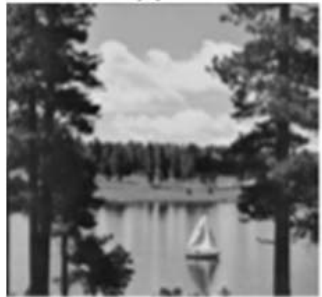

(c)

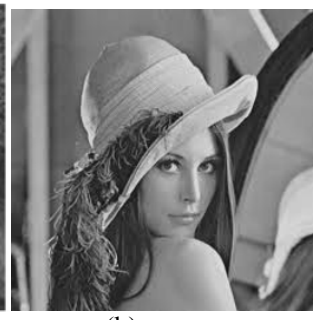

(b)

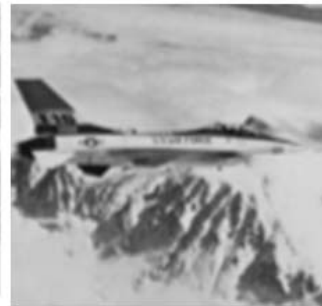

(d)

Fig.5 Host images (a) Baboon, (b) Lena (c) Lake (d) Plane

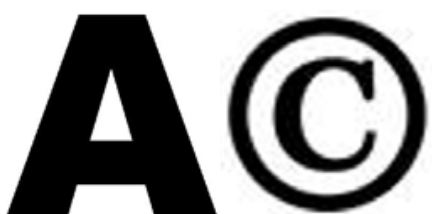

(a)

(b)

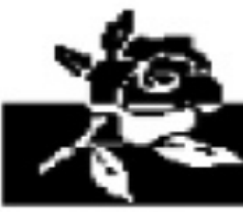

(c)

Fig.6 Watermark images (a) Character (b) Logo (c) Rose 
To evaluate the performance of proposed approach, four perfomrance metrics such as Mean Square Error (MSE), Peak Signal to Noise Ratio (PSNR), Normalized Correlation (NC) and Structural Similarity Index Measure (SSIM) were considered and the respective mathematical formulation is given as,

$M S E=\frac{1}{M X N} \sum_{i=1}^{M} \sum_{j=1}^{N}\left(w(i, j)-w^{*}(i, j)\right)^{2}(20)$

Where

$w=$ original watermark image

$w^{*}=$ extracted watermark image

$$
\begin{aligned}
& P S N R=10 * \log \left(255^{2} / M S E\right) \\
& N C=\sum_{i=1}^{M} \sum_{j=1}^{N} \frac{w(i, j) * w^{*}(i, j)}{(w(i, j))^{2} * w^{*}((i, j))^{2}} \\
& S S I M=\frac{\sum_{i} \sum_{i} w(i, j) \otimes w^{*}(i, j)}{\sum_{i} \sum_{i}(w(i, j))^{2}}
\end{aligned}
$$

The NC is also used for the evaluation of fitness function of PSO. The fitness function of PSO is defined as fitness $\left(s_{j}\right)=1-\operatorname{Average}\left(N C_{j}\right)$ $N C_{j}=\frac{1}{n_{\text {attack }}} \sum_{k=1}^{n_{\text {attack }}} N C\left(w, w_{j}^{*, k}\right)$

Where $w_{j}^{*, k}$ represents the extracted watermark through the proposed approach characterized by the position of the $\mathrm{j}_{\text {th }}$ particle. The smaller fitness value means the better robustness. Let, $n_{\text {attack }}$ signifies the number of attacks, here the $n_{\text {attack }}$ is set to 8 . Because, totally eight types of attacks are simulated in the simulation.

Performance metrics was evaluated for both No attack and Attack scenarios. At both phases, the proposed approach was compared with T. Naheed et.al [6] and S.P.Rao et.al[41].

A. No attack scenario

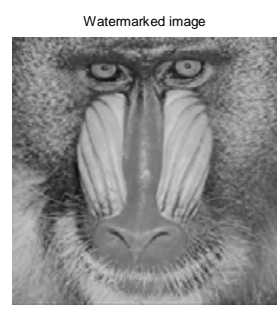

(a)

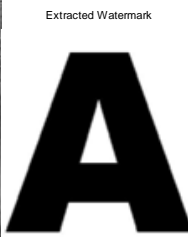

(b)

Fig.7. (a) watermarked image (b)exrated watermark image

\section{B. Attack Scenario}

1. Gaussian Noise Attack (variance $=0.001,0.01,0.1$ )

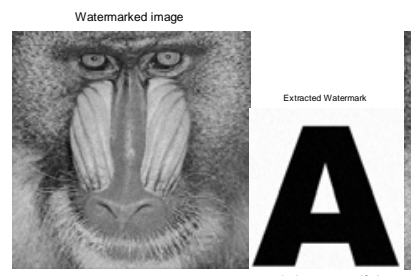

(a)

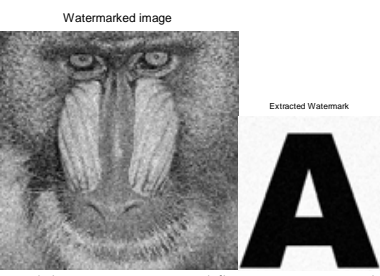

(d)

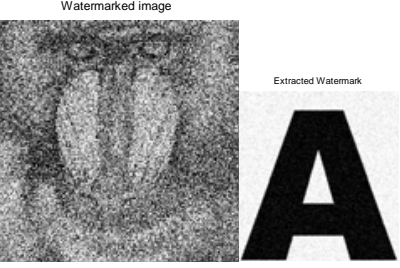

(f)

Fig.8. Obtained results in the case of gaussian noise attack scenario

2. Salt \& Pepper Noise Attack (variance $=0.001,0.01,0.1$ )

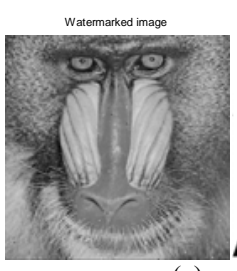

(a)

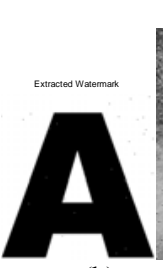

(b)

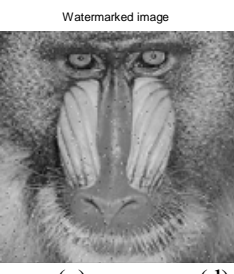

(c)

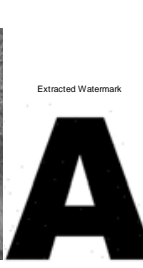

(d)

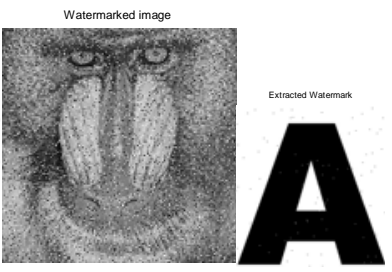

(f)

Fig.9. Obtained results in the case of salt $\&$ pepper noise attack scenario 
3. Median Filtering Attack of average size $3 X 3$ and $7 X 7$

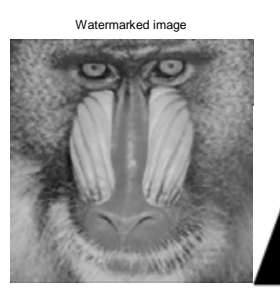

(a)

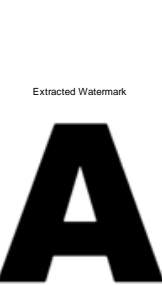

(b)

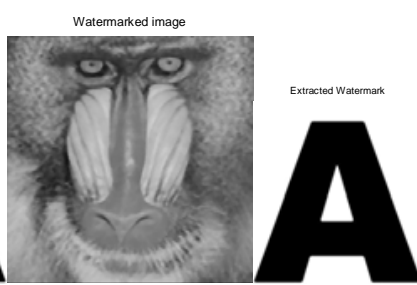

(c)

(d)

Fig.10. Obtained results in the case of Median Filtering attack scenario

4. Histogram Equalization Attack

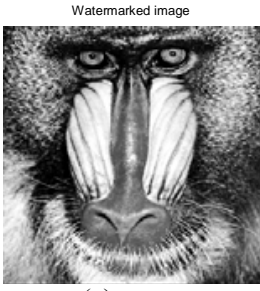

(a)

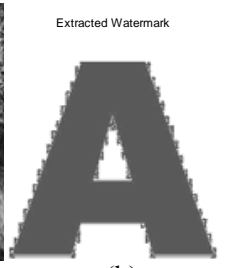

(b)

Fig.11. Obtained results in the case of Histogram Equalization attack scenario

5. Rotation Attack at $30^{\circ}$ and $45^{\circ}$ )
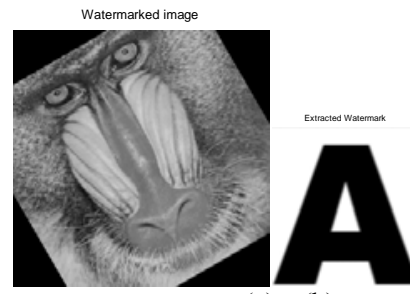

(a) (b)

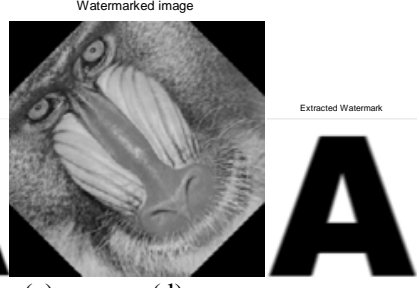

(d)

Fig.12. Obtained results in the case of Rotation attack scenario

6. Constrast Enhancemnet Attack at contrast limit $=0.03$ and 0.05

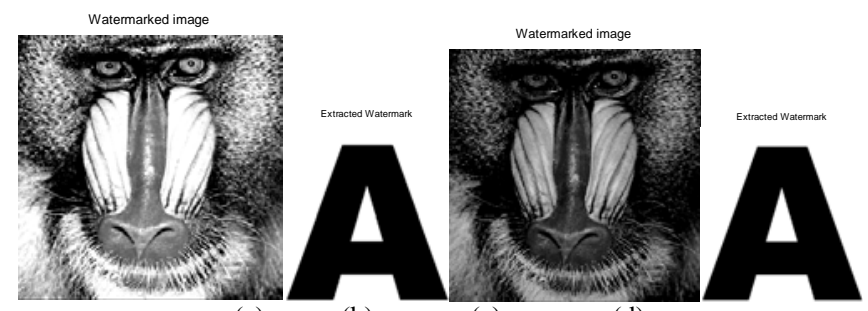

(a)

(b)

(c)

(d)

Fig.13. Obtained results in the case of Contrast Enhancemnet attack scenario

7. Cropping Attack

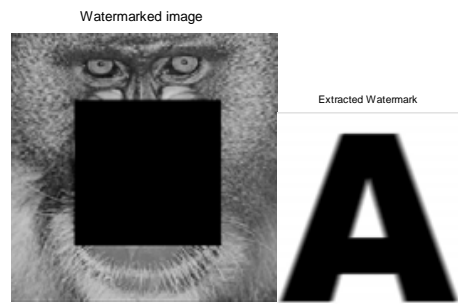

(a)

(b)

Fig.14. Obtained results in the case of Cropping attack scenario 
8. Scalling Attack at nearest neighbor, bi-linear and bi-cubic

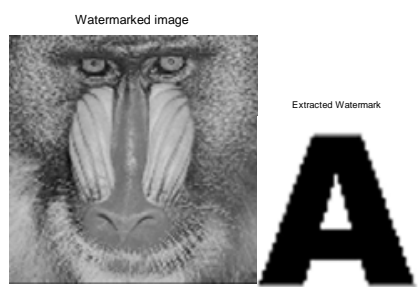

(a)

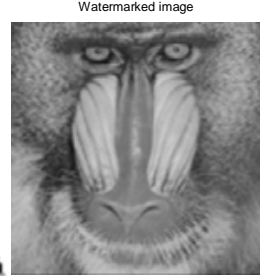

(b) (c)

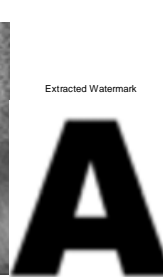

(d) (e)

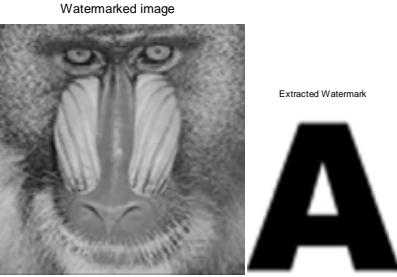

(f)

Fig.15. Obtained results in the case of scalling attack scenario

The evaluated PSNR, MSE, NC and SSIM of all the attack scenarios are represented in the following tables.

Table. 1. Performance analysis of proposed approach in the case of no attack scenario

\begin{tabular}{|c|c|c|c|c|c|c|}
\hline Metrics & \multicolumn{3}{|c|}{ Baboon with character } & \multicolumn{3}{c|}{ Lena with Logo } \\
\hline & $\begin{array}{c}\text { S.P.Rao.et.al } \\
\text { [41] }\end{array}$ & T.N.et.al [6] & Proposed & S.P.Rao.et.al [41] & T.N.et.al [6] & Proposed \\
\hline PSNR & 47.9936 & 48.5514 & 52.7485 & 48.8982 & 49.2232 & 53.0287 \\
\hline MSE & 1.0321 & 0.9077 & 0.3453 & 0.8380 & 0.7776 & 0.3238 \\
\hline NC & 0.9789 & 0.9825 & 0.9869 & 0.9812 & 0.9832 & 0.9876 \\
\hline SSIM & 0.9824 & 0.9857 & 0.9892 & 0.9842 & 0.9858 & 0.9883 \\
\hline
\end{tabular}

Table.2. Performance analysis of proposed approach for gaussian noise attack while varying noise variance

\begin{tabular}{|c|c|c|c|c|c|c|c|}
\hline Variance & Metrics & \multicolumn{3}{|c|}{ Baboon with character } & \multicolumn{3}{c|}{ Lena with Logo } \\
\hline \multirow{3}{*}{0.001} & & $\begin{array}{c}\text { S.P.Rao.et.al } \\
\text { [41] }\end{array}$ & T.N.et.al [6] & Proposed & $\begin{array}{c}\text { S.P.Rao.et.al } \\
\text { [41] }\end{array}$ & T.N.et.al [6] & Proposed \\
& PSNR & 47.7512 & 48.1418 & 50.9879 & 47.9985 & 48.6698 & 51.2275 \\
\cline { 2 - 8 } & MSE & 1.0913 & 0.9975 & 0.5180 & 1.0309 & 0.8833 & 0.4902 \\
\cline { 2 - 8 } & NC & 0.8998 & 0.9012 & 0.9119 & 0.9005 & 0.9196 & 0.9238 \\
\cline { 2 - 8 } & SSIM & 0.9644 & 0.9688 & 0.9785 & 0.9648 & 0.9698 & 0.9798 \\
\hline \multirow{4}{*}{0.01} & PSNR & 47.2276 & 47.4478 & 50.2041 & 47.3325 & 48.3363 & 50.8689 \\
\cline { 2 - 8 } & MSE & 1.2312 & 1.1703 & 0.6204 & 1.2018 & 0.9538 & 0.5323 \\
\cline { 2 - 8 } & NC & 0.8845 & 0.8898 & 0.9069 & 0.8812 & 0.8856 & 0.9172 \\
\cline { 2 - 8 } & SSIM & 0.9628 & 0.9637 & 0.9735 & 0.9621 & 0.9645 & 0.9766 \\
\hline \multirow{6}{*}{0.1} & PSNR & 46.9898 & 47.0549 & 49.9338 & 47.0201 & 48.1298 & 50.0371 \\
\cline { 2 - 8 } & MSE & 1.3005 & 1.2811 & 0.6602 & 1.2914 & 1.0002 & 0.6647 \\
\cline { 2 - 8 } & NC & 0.8797 & 0.8835 & 0.9013 & 0.8809 & 0.8824 & 0.9022 \\
\cline { 2 - 8 } & SSIM & 0.9583 & 0.9615 & 0.9638 & 0.9606 & 0.9632 & 0.9721 \\
\hline
\end{tabular}

Table.3. Performance analysis of proposed approach for salt $\&$ pepper noise attack while varying noise variance

\begin{tabular}{|c|c|c|c|c|c|c|c|}
\hline Variance & Metrics & \multicolumn{3}{|c|}{ Baboon with character } & \multicolumn{3}{c|}{ Lena with Logo } \\
\hline \multirow{4}{*}{0.001} & & $\begin{array}{c}\text { S.P.Rao.et.al } \\
\text { [41] }\end{array}$ & T.N.et.al [6] & Proposed & $\begin{array}{c}\text { S.P.Rao.et.al } \\
\text { [41] }\end{array}$ & T.N.et.al [6] & Proposed \\
& PSNR & 46.7473 & 47.2536 & 49.8754 & 46.8891 & 47.7471 & 49.9963 \\
\cline { 2 - 8 } & MSE & 1.3752 & 1.2238 & 0.6692 & 1.3310 & 1.0924 & 0.6508 \\
\cline { 2 - 8 } & NC & 0.9618 & 0.9635 & 0.9685 & 0.9635 & 0.9655 & 0.9681 \\
\cline { 2 - 8 } & SSIM & 0.9456 & 0.9489 & 0.9519 & 0.9486 & 0.9498 & 0.9522 \\
\hline \multirow{4}{*}{0.01} & PSNR & 46.2234 & 46.8481 & 49.0286 & 46.3814 & 47.1789 & 49.3399 \\
\cline { 2 - 8 } & MSE & 1.5515 & 1.3436 & 0.8132 & 1.4960 & 1.2451 & 0.7570 \\
\cline { 2 - 8 } & NC & 0.9601 & 0.9624 & 0.9646 & 0.9585 & 0.9623 & 0.9651 \\
\cline { 2 - 8 } & SSIM & 0.9417 & 0.9456 & 0.9490 & 0.9422 & 0.9466 & 0.9507 \\
\hline \multirow{6}{*}{0.1} & PSNR & 45.8746 & 46.1325 & 48.2358 & 46.1238 & 46.8576 & 48.8339 \\
\cline { 2 - 8 } & MSE & 1.6812 & 1.5843 & 0.9761 & 1.5835 & 1.3407 & 0.8505 \\
\cline { 2 - 8 } & NC & 0.9519 & 0.9559 & 0.9573 & 0.9515 & 0.9565 & 0.9595 \\
\cline { 2 - 8 } & SSIM & 0.9367 & 0.9396 & 0.9420 & 0.9369 & 0.9402 & 0.9469 \\
\hline
\end{tabular}

Table.4. Performance analysis of proposed approach for Median Filtering attack scenario with varying size

\begin{tabular}{|c|c|c|c|c|c|c|c|}
\hline Variance & Metrics & \multicolumn{3}{|c|}{ Baboon with character } & \multicolumn{3}{c|}{ Lena with Logo } \\
\hline & & $\begin{array}{c}\text { S.P.Rao.et.al } \\
\text { [41] }\end{array}$ & T.N.et.al [6] & Proposed & $\begin{array}{c}\text { S.P.Rao.et.al } \\
\text { [41] }\end{array}$ & T.N.et.al [6] & Proposed \\
\hline \multirow{3}{*}{3 X3 } & PSNR & 47.1015 & 47.5589 & 48.5680 & 47.2021 & 47.8680 & 48.7990 \\
\cline { 2 - 8 } & MSE & 1.2674 & 1.1407 & 0.9042 & 1.2384 & 1.0624 & 0.8574 \\
\cline { 2 - 8 } & NC & 0.9555 & 0.9569 & 0.9589 & 0.9563 & 0.9577 & 0.9586 \\
\cline { 2 - 8 } & SSIM & 0.9383 & 0.9399 & 0.9478 & 0.9386 & 0.9419 & 0.9461 \\
\hline \multirow{3}{*}{7 X7 } & PSNR & 46.5312 & 47.1013 & 48.1090 & 46.3158 & 47.1519 & 48.3960 \\
\cline { 2 - 8 } & MSE & 1.4453 & 1.2675 & 1.0050 & 1.5188 & 1.2528 & 0.9408 \\
\cline { 2 - 8 } & NC & 0.9499 & 0.9533 & 0.9542 & 0.9493 & 0.9528 & 0.9567 \\
\cline { 2 - 8 } & SSIM & 0.9255 & 0.9267 & 0.9301 & 0.9229 & 0.9296 & 0.9355 \\
\hline
\end{tabular}


Table.5. Performance analysis of proposed approach for Histogram Equalization attack

\begin{tabular}{|c|c|c|c|c|c|c|}
\hline Metrics & \multicolumn{3}{|c|}{ Baboon with character } & \multicolumn{3}{c|}{ Lena with Logo } \\
\hline & $\begin{array}{c}\text { S.P.Rao.et.al } \\
\text { [41] }\end{array}$ & T.N.et.al [6] & Proposed & S.P.Rao.et.al [41] & T.N.et.al [6] & Proposed \\
\hline PSNR & 27.6349 & 29.3685 & 31.9785 & 27.2241 & 29.2247 & 31.8555 \\
\hline MSE & 112.0961 & 75.2022 & 41.2316 & 123.2169 & 77.7329 & 42.4161 \\
\hline NC & 0.8839 & 0.8869 & 0.8992 & 0.8823 & 0.8855 & 0.8986 \\
\hline SSIM & 0.9317 & 0.9346 & 0.9379 & 0.9274 & 0.9327 & 0.9366 \\
\hline
\end{tabular}

Table.6. Performance analysis of proposed approach for Rotation attack scenario with varying angle of rotation

\begin{tabular}{|c|c|c|c|c|c|c|c|}
\hline Rotation & Metrics & \multicolumn{3}{|c|}{ Baboon with character } & \multicolumn{3}{c|}{ Lena with Logo } \\
\hline & & $\begin{array}{c}\text { S.P.Rao.et.al } \\
\text { [41] }\end{array}$ & T.N.et.al [6] & Proposed & $\begin{array}{c}\text { S.P.Rao.et.al } \\
\text { [41] }\end{array}$ & T.N.et.al [6] & Proposed \\
\hline \multirow{3}{*}{$30^{0}$} & PSNR & 22.3963 & 24.5689 & 30.3252 & 21.5335 & 23.3312 & 30.1128 \\
\cline { 2 - 8 } & MSE & 374.49 & 227.68 & 60.3338 & 456.80 & 301.96 & 63.3578 \\
\cline { 2 - 8 } & NC & 0.8748 & 0.8965 & 0.9245 & 0.8797 & 0.8814 & 0.9287 \\
\cline { 2 - 8 } & SSIM & 0.8819 & 0.8956 & 0.9328 & 0.8831 & 0.8844 & 0.9338 \\
\hline \multirow{4}{*}{$45^{0}$} & PSNR & 22.2285 & 24.4578 & 30.1285 & 21.3388 & 23.1102 & 30.0360 \\
\cline { 2 - 8 } & MSE & 389.25 & 232.97 & 63.1292 & 477.74 & 317.73 & 64.4882 \\
\cline { 2 - 8 } & NC & 0.8652 & 0.8766 & 0.9222 & 0.8723 & 0.8786 & 0.9254 \\
\cline { 2 - 8 } & SSIM & 0.8796 & 0.8823 & 0.9313 & 0.8744 & 0.8794 & 0.9321 \\
\hline
\end{tabular}

Table.7. Performance analysis of proposed approach in the case of Contrast Enhancemnet attack with varying contrast limit

\begin{tabular}{|c|c|c|c|c|c|c|c|}
\hline Limit & Metrics & \multicolumn{3}{|c|}{ Baboon with character } & \multicolumn{3}{c|}{ Lena with Logo } \\
\hline & & $\begin{array}{c}\text { S.P.Rao.et.al } \\
\text { [41] }\end{array}$ & T.N.et.al [6] & Proposed & $\begin{array}{c}\text { S.P.Rao.et.al } \\
\text { [41] }\end{array}$ & T.N.et.al [6] & Proposed \\
\hline \multirow{4}{*}{0.03} & PSNR & 30.2145 & 31.7728 & 32.7463 & 29.6336 & 31.9696 & 32.8389 \\
\cline { 2 - 8 } & MSE & 61.8914 & 43.2315 & 34.5502 & 70.7490 & 41.3162 & 33.8213 \\
\cline { 2 - 8 } & NC & 0.9247 & 0.9278 & 0.9293 & 0.9298 & 0.9322 & 0.9356 \\
\cline { 2 - 8 } & SSIM & 0.9566 & 0.9599 & 0.9647 & 0.9589 & 0.9636 & 0.9670 \\
\hline \multirow{3}{*}{0.05} & PSNR & 29.9985 & 30.2252 & 31.9697 & 28.9631 & 30.0028 & 31.9492 \\
\cline { 2 - 8 } & MSE & 65.0475 & 61.7391 & 41.3152 & 82.5601 & 64.9831 & 41.5107 \\
\cline { 2 - 8 } & NC & 0.9183 & 0.9196 & 0.9277 & 0.9189 & 0.9245 & 0.9281 \\
\cline { 2 - 8 } & SSIM & 0.9537 & 0.9587 & 0.9623 & 0.9522 & 0.9629 & 0.9658 \\
\hline
\end{tabular}

Table.8. Performance analysis of proposed approach for cropping attack

\begin{tabular}{|c|c|c|c|c|c|c|}
\hline Metrics & \multicolumn{3}{|c|}{ Baboon with character } & \multicolumn{3}{c|}{ Lena with Logo } \\
\hline & $\begin{array}{c}\text { S.P.Rao.et.al } \\
\text { [41] }\end{array}$ & T.N.et.al [6] & Proposed & S.P.Rao.et.al [41] & T.N.et.al [6] & Proposed \\
\hline PSNR & 19.8634 & 21.6631 & 24.5789 & 18.8524 & 21.0129 & 24.1329 \\
\hline MSE & 671.02 & 443.37 & 226.56 & 846.91 & 514.98 & 251.06 \\
\hline NC & 0.7285 & 0.7358 & 0.7449 & 0.7238 & 0.7339 & 0.7396 \\
\hline SSIM & 0.8125 & 0.8229 & 0.8494 & 0.8093 & 0.8156 & 0.8379 \\
\hline
\end{tabular}

Table.9. Performance analysis of proposed approach for scalling attack scenario with various scalling techniques

\begin{tabular}{|c|c|c|c|c|c|c|c|}
\hline $\begin{array}{c}\text { Scaling } \\
\text { technique }\end{array}$ & Metrics & \multicolumn{3}{|c|}{ Baboon with character } & \multicolumn{3}{c|}{ Lena with Logo } \\
\hline & & $\begin{array}{c}\text { S.P.Rao.et.al } \\
{[\mathbf{4 1 1}}\end{array}$ & T.N.et.al [6] & Proposed & $\begin{array}{c}\text { S.P.Rao.et.al } \\
\text { [41] }\end{array}$ & T.N.et.al [6] & Proposed \\
\hline \multirow{3}{*}{$\begin{array}{c}\text { Nearest } \\
\text { neighor }\end{array}$} & PSNR & 32.9721 & 33.2147 & 37.3339 & 32.3396 & 32.8985 & 37.2327 \\
\cline { 2 - 8 } & MSE & 32.7997 & 31.0178 & 12.0141 & 37.9420 & 33.3603 & 12.2973 \\
\cline { 2 - 8 } & NC & 0.9641 & 0.9668 & 0.9721 & 0.9655 & 0.9673 & 0.9682 \\
\cline { 2 - 8 } & SSIM & 0.9613 & 0.9638 & 0.9749 & 0.9647 & 0.9680 & 0.9687 \\
\hline \multirow{3}{*}{ Bi-linear } & PSNR & 34.6685 & 34.9967 & 38.4418 & 34.2285 & 35.2287 & 38.3387 \\
\cline { 2 - 8 } & MSE & 22.1937 & 20.5783 & 9.3089 & 24.5601 & 19.5079 & 9.5326 \\
\cline { 2 - 8 } & NC & 0.9688 & 0.9699 & 0.9133 & 0.9695 & 0.9719 & 0.9138 \\
\cline { 2 - 8 } & SSIM & 0.9633 & 0.9683 & 0.9758 & 0.9698 & 0.9732 & 0.9757 \\
\hline \multirow{3}{*}{ Bi-cubi } & PSNR & 36.2215 & 36.5878 & 39.6321 & 36.1328 & 36.3367 & 39.2124 \\
\cline { 2 - 8 } & MSE & 15.5214 & 14.2659 & 7.0955 & 15.8416 & 15.1151 & 7.7954 \\
\cline { 2 - 8 } & NC & 0.9731 & 0.9751 & 0.9766 & 0.9721 & 0.9736 & 0.9765 \\
\cline { 2 - 8 } & SSIM & 0.9745 & 0.9772 & 0.9784 & 0.9788 & 0.9790 & 0.9794 \\
\hline
\end{tabular}

A case study was carried out by considering the charcater image as watermark and the Baboon image as host. The obtained PSNR, NC and SSIM are represented in the figure.16. Similarly, a one more case study is carried out for Logo and Lena as watermark and host images. The obatined results are shown in figure.17. 

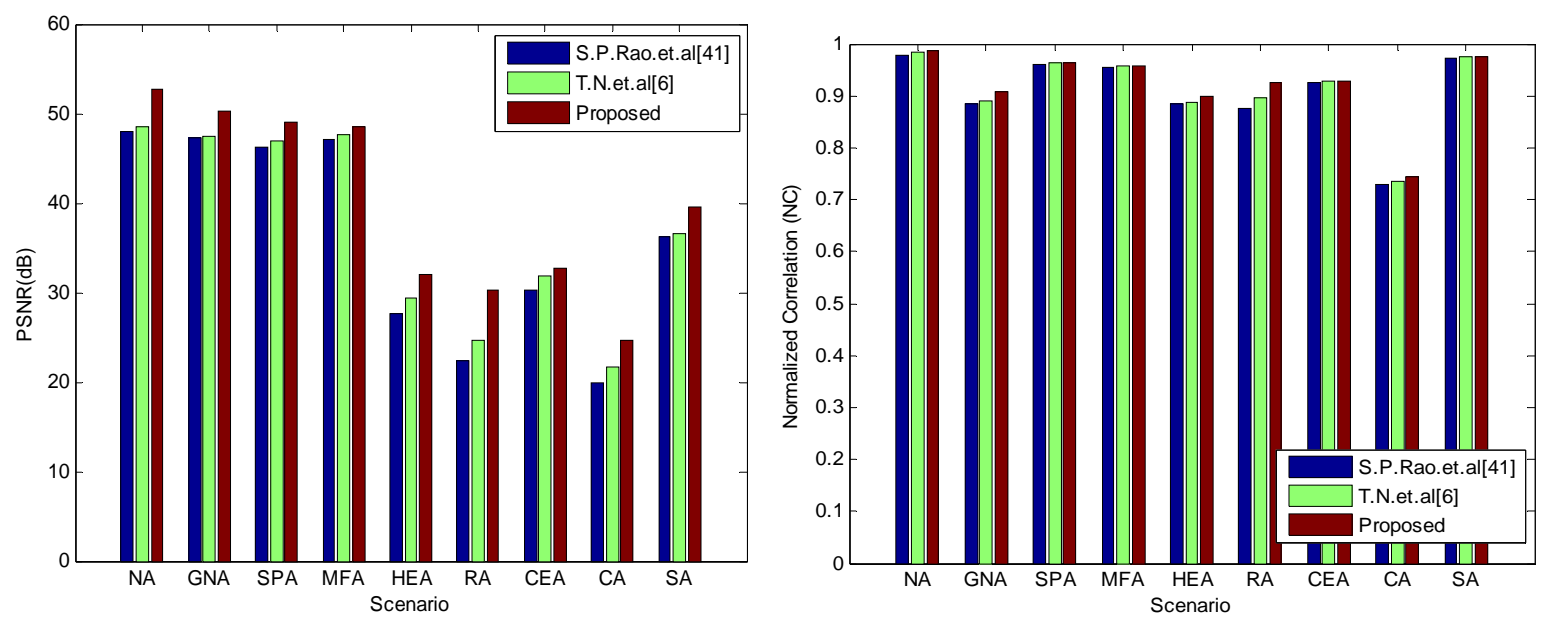

(a)(b)

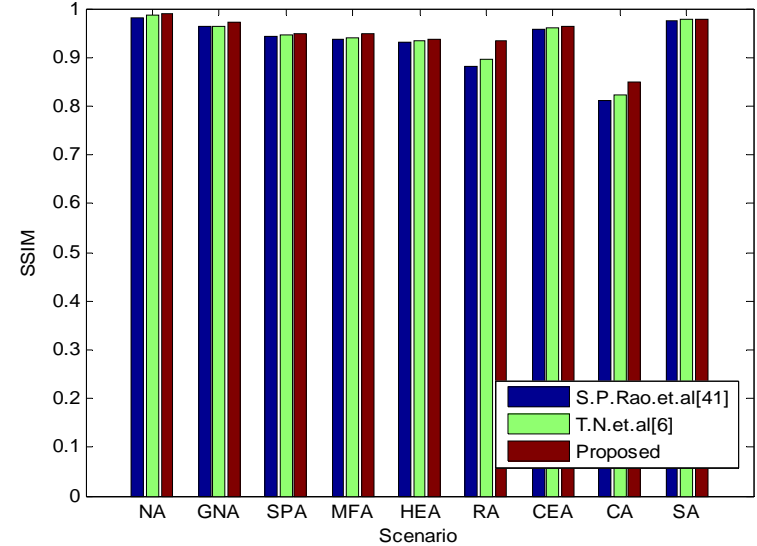

(c)

Fig.16. Performance metrics for the test case of Baboon image as a host and Character image as a watermark (a) PSNR, (b) NC and (c) SSIM
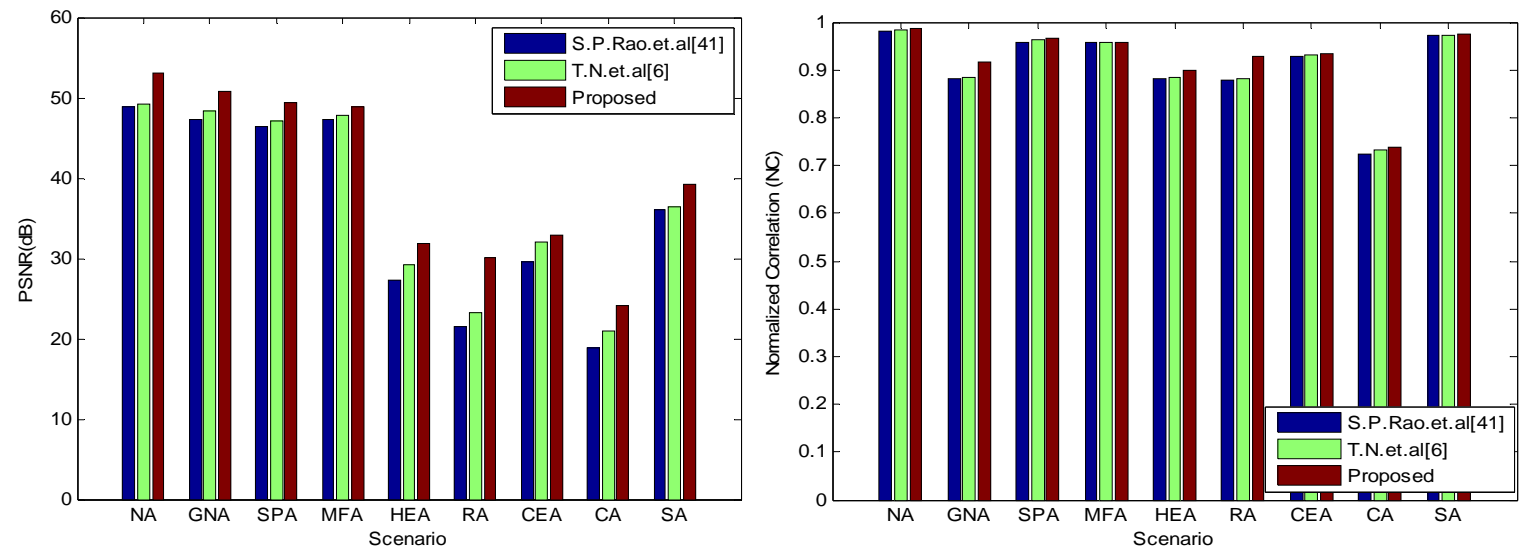

(a)(b) 


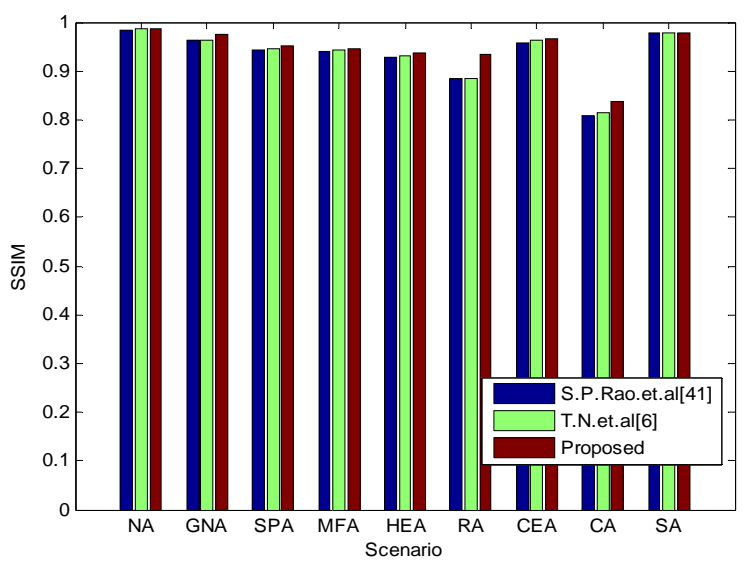

(c)

Fig.17. Performance metrics for the test case of Lena image as a host and Logo image as a watermark (a) PSNR, (b) NC and (c) SSIM

Another caste study is performed over convnetional and proposed approaches by varying the noise variance over gaussian noise attack and the obtained PSNR, NC and SSIM are represented in figures.18. In this case the Lena image is considered as host and the Rose image is considered as watermark.
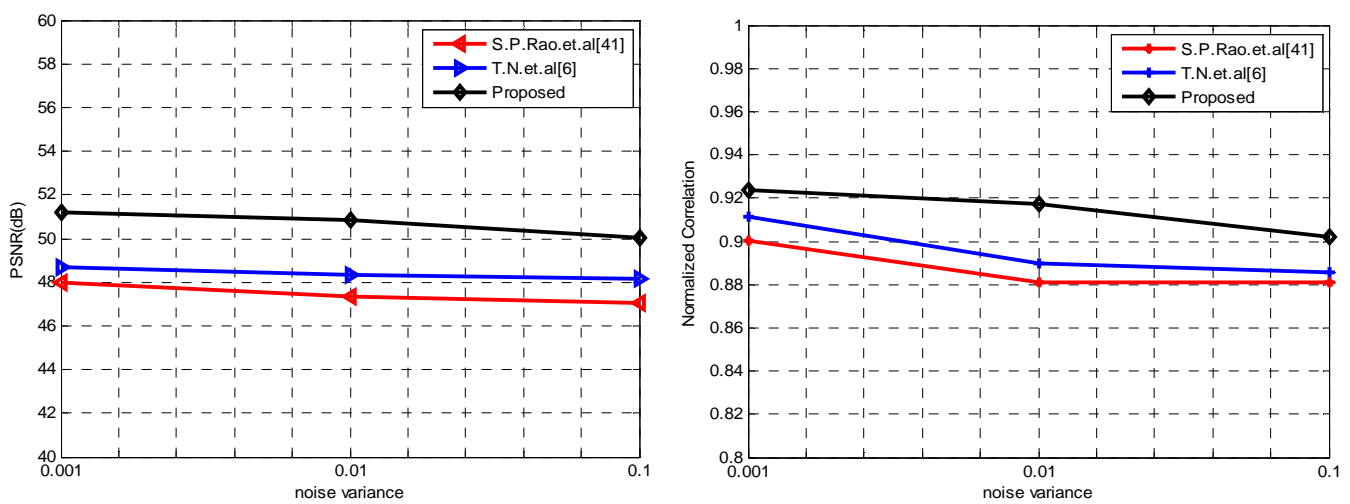

(a)(b)

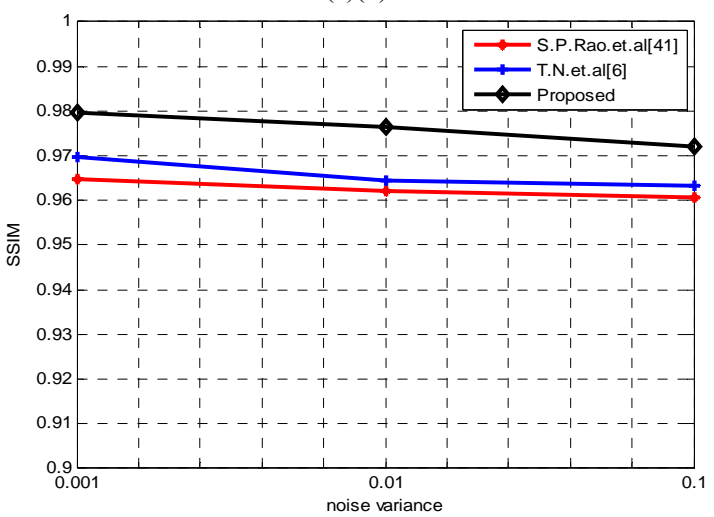

(c)

Fig.18. Performance metrics for the test case of Lena image as a host and Logo image as a watermark for varying gaussian noise variance (a) PSNR, (b) NC and (c) SSIM

Form the fig.18(a), it can observed that the PSNR is gradually decreaisng with increasing nois evariance. At every instant of noise variance, the proposed approach is observed to be an efficient, because the proposed approach obtained an increaesd PSNR compared to cnvnetinal approaches. Similarly, fig.18 (b) and fig.18(c) elevtaes the robustness of proposed approach with respect to NC and SSIM.

The performance of watermarking scheme will vary with the noise varinace under the salt \& pepper noise attack also. The noise varinace is varied as $0.001,0.01$ and 0.1 and the obtained PSNR, NC and SSIM considering the Lena as a host and Logo as a watermark is represenetd in the figure.19. 

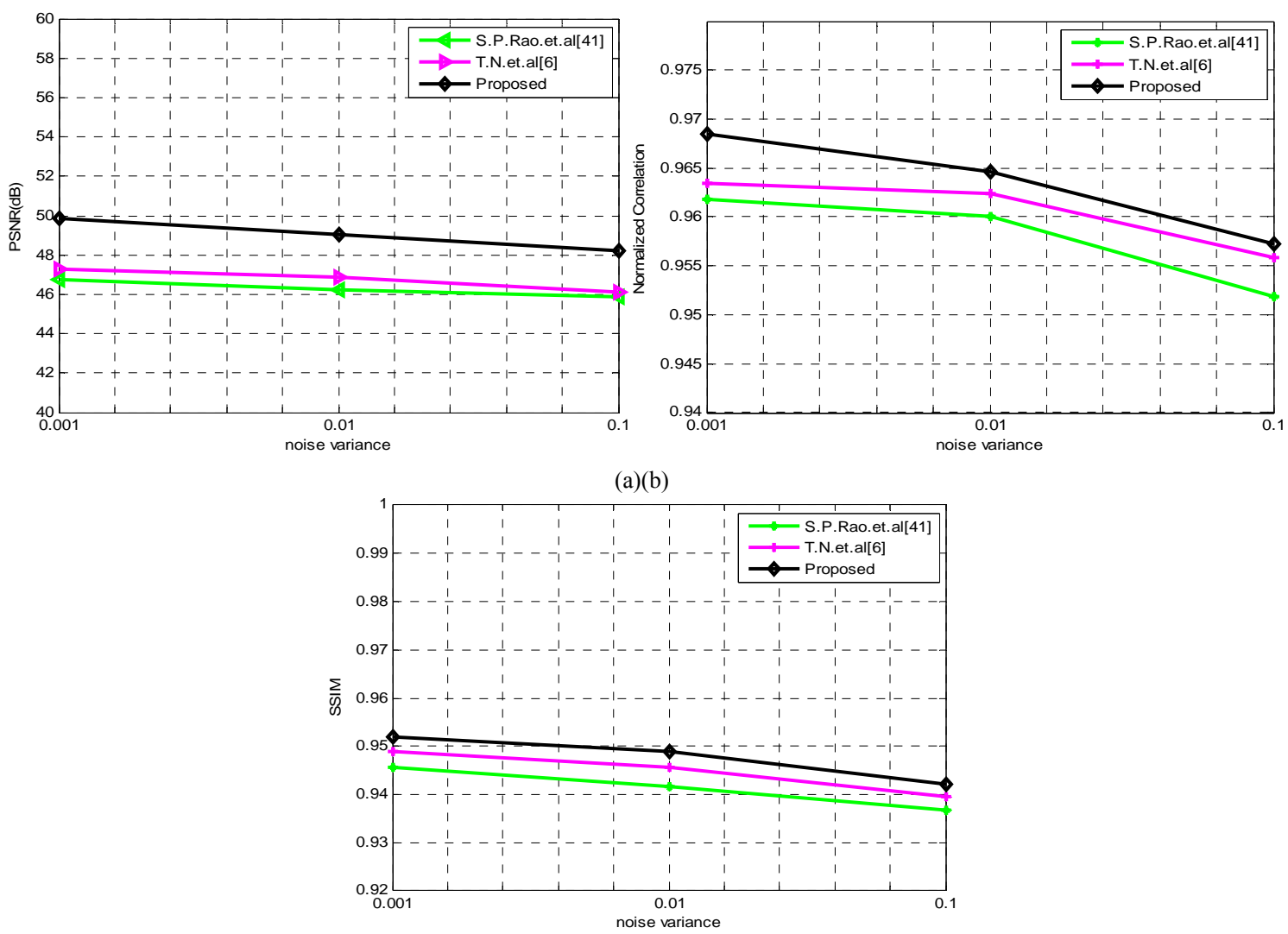

(c)

Fig.19. Performance metrics for the test case of Lena image as a host and Logo image as a watermark for varying salt \& pepper noise variance (a) PSNR, (b) NC and (c) SSIM

From the above performance analysis, it can be observed that the proposed approach showing a superior performance over conventional approaches in all cases. It is obseved that the proposed approach shown an excellent performance in the case of rotation attack. In that rotation attack scenario, the proposed approach achieved an improved PSNR of 4-5dB on an average.

\section{CONCLUSION}

In this paper, a new watermarking approach was proposed to enhance the robustness in various applications and also to ensure the imperceptibility. A combination of artificial intelligent techniques such as GA and PSO were utilized here to improve the performance. In this paper, the GA is accomplished at the feature extraction phase to find the optimum features which are roust for any type of attacks and PSO is used for enhancing the strength of watermark process. Along with these methods, at preprocessing stage, the original host image is subjected to a normalization process, particularly to obtain the features which are invariant. Then the further process is accomplished on the obtained invariant features. This normalization increases the robustness of watermarking approach in the case or rotation attack specifically. Finally, a performance evaluation is carried out by considering various host and watermark images and the performance metrics PSNR, MSE, NC and SSIM were evaluated to verify the proposed approach. A comparative analysis is also carried out by comparing the proposed approach with conventional approaches at various cases and proved as the proposed approach is robust. Thus, the proposed scheme has satisfied the robustness, and imperceptibility, requirements that are essential for a robust watermarking scheme.

\section{REFERENCES}

[1] I. J. Cox, J. Kilian, F. T. Leighton, and T. Shamoon, "Secure spread spectrum watermarking for multimedia," IEEE Transactions on Image Processing, vol. 6, no. 12, pp. 1673-1687, 1997.

[2] R. Liu and T. Tan, "An SVD-based watermarking scheme for protecting rightful ownership," IEEE Transactions on Multimedia, vol. 4, no. 1 , pp. 121-128, 2002.

[3] M. L. Miller, I. J. Cox, J.-P. M. G. Linnartz, and T. Kalker, "A review of watermarking principles and practices," in Digital Signal Processing in Multimedia Systems, pp. 461-485, 1999.

[4] E. Hussein and M. A. Belal, "Digital watermarking techniques, applications and attacks applied to digital media: a survey," International Journal of Engineering Research and Technology, vol. 1, no. 7, pp. 1-8, 2012.

[5] A. Khan, A. Siddiqa, S.Munib, and S. A.Malik, "A recent survey of reversible watermarking techniques," Information Sciences, vol. 279, pp. 251-272, 2014.

[6] Talat Naheed, "Intelligent reversible watermarking technique in medical images using GA and PSO", Optik - Int. J. Light Electron, 2014. 
[7] Asha Rani, “A Zero-Watermarking Scheme using Discrete Wavelet Transform”, in Proc., of International Conference on Eco-friendly Computing and Communication Systems (ICECCS), 2015.

[8] L.-D. Li and B.-L. Guo, "Localized image watermarking in spatial domain resistant to geometric attacks," AEU: International Journal of Electronics and Communications, vol. 63, no. 2, pp. 123-131, 2009.

[9] Q. Su, Y. Niu, Q. Wang, and G. Sheng, "A blind color image watermarking based on DC component in the spatial domain," Optik, vol. 124, no. 23, pp. 6255-6260, 2013.

[10] C.-H. Yang, C.-Y. Weng, S.-J. Wang, and H.-M. Sun, "Adaptive data hiding in edge areas of images with spatial LSB domain systems," IEEE Transactions on Information Forensics and Security, vol. 3, no. 3, pp. 488-497, 2008

[11] S. S. Jamal, T. Shah, and I. Hussain, "An efficient scheme for digital watermarking using chaotic map," Nonlinear Dynamics, vol. 73, no. 3, pp. 1469-1474, 2013.

[12] S. D. Lin, S.-C. Shie, and J. Y. Guo, "Improving the robustness of DCT-based image watermarking against JPEG compression," Computer Standards and Interfaces, vol. 32, no. 1-2, pp. 54-60, 2010.

[13] B. Chen, G. Coatrieux, G. Chen, X. Sun, J. L. Coatrieux, and H. Shu, "Full 4-D quaternion discrete Fourier transform based watermarking for color images," Digital Signal Processing, vol. 28, no. 1, pp. 106-119, 2014.

[14] J. Lang and Z.-G. Zhang, "Blind digital watermarking method in the fractional Fourier transform domain," Optics and Lasers in Engineering, vol. 53, pp. 112-121, 2014.

[15] E. H. Elshazly, O. S. Faragallah, A. M. Abbas, "Robust and secure fractional wavelet image watermarking," Signal, Image and Video Processing, Vol.9, pp.89-98, December 2015.

[16] L. Li, H.-H. Xu, C.-C. Chang, and Y.-Y. Ma, "A novel image watermarking in redistributed invariant wavelet domain," Journal of Systems and Software, vol. 84, no. 6, pp. 923-929, 2011.

[17] N. Liu,H. Li, H. Dai, D. Guo, andD.Chen, "Robust blind image watermarking based on chaotic mixtures," Nonlinear Dynamics, vol. 80, no. 3, pp. 1329-1355, 2015.

[18] M.-Q. Fan, H.-X. Wang, and S.-K. Li, "Restudy on SVD-based watermarking scheme," Applied Mathematics and Computation, vol. 203, no. 2, pp. 926-930, 2008.

[19] A. A.Mohammad, A. Alhaj, and S. Shaltaf, "An improved SVD based watermarking scheme for protecting rightful ownership," Signal Processing, vol. 88, no. 9, pp. 2158-2180, 2008.

[20] Q. Su, Y. Niu, H. Zou, and X. Liu, "A blind dual color images watermarking based on singular value decomposition," Applied Mathematics and Computation, vol. 219, no. 16, pp. 8455-8466, 2013.

[21] H.-T. Hu and L.-Y. Hsu, "Exploring DWT-SVD-DCT feature parameters for robust multiple watermarking against JPEG and JPEG2000 compression,” Computers and Electrical Engineering, vol. 41, pp. 52-63, 2015.

[22] N. M.Makbol and B. E. Khoo, "Robust blind image watermarking scheme based on Redundant Discrete Wavelet Transform and Singular Value Decomposition,” AEU—International Journal of Electronics and Communications, vol. 67,no. 2, pp. 102-112, 2013.

[23] N. M.Makbol and B. E. Khoo, "A new robust and secure digital image watermarking scheme based on the integer wavelet transform and singular value decomposition," Digital Signal Processing, vol. 33, pp. 134-147, 2014.

[24] X. Wu and W. Sun, "Robust copyright protection scheme for digital images using overlapping DCT and SVD," Applied Soft Computing Journal, vol. 13, no. 2, pp. 1170-1182, 2013.

[25] P.-P. Zheng, J. Feng, Z. Li, and M.-Q. Zhou, "A novel SVD and LS-SVM combination algorithm for blind watermarking," Neurocomputing, vol. 142, pp. 520-528, 2014.

[26] A. Mishra, C. Agarwal, A. Sharma, and P. Bedi, "Optimized gray-scale image watermarking using DWT-SVD and Firefly Algorithm," Expert Systems with Applications, vol. 41, no. 17, pp. 7858-7867, 2014.

[27] M. Ali and C.W. Ahn, "An optimized watermarking technique based on self-adaptive de in DWT-SVD transform domain," Signal Processing, vol. 94, no. 1, pp. 545-556, 2014.

[28] M. Ali, C. W. Ahn, and M. Pant, "A robust image watermarking technique using SVD and differential evolution in DCT domain," Optik, vol. 125, no. 1, pp. 428-434, 2014.

[29] M. Ali, C.W. Ahn, M. Pant, and P. Siarry, "An image watermarking scheme in wavelet domain with optimized compensation of singular value decomposition via artificial bee colony," Information Sciences, vol. 301, pp. 44-60, 2015.

[30] M. Ali, C. W. Ahn, and P. Siarry, "Differential evolution algorithm for the selection of optimal scaling factors in image watermarking," Engineering Applications of Artificial Intelligence, vol. 31, pp. 15-26, 2014.

[31] S. H. Amiri and M. Jamzad, "Robust watermarking against print and scan attack through efficient modeling algorithm," Signal Processing: Image Communication, vol. 29, no. 10, pp. 1181-1196, 2014.

[32] B. Lei, D. Ni, S. Chen, T. Wang, and F. Zhou, "Optimal image watermarking scheme based on chaotic map and quaternion wavelet transform,” Nonlinear Dynamics, vol. 78, no. 4, pp. 2897-2907, 2014.

[33] R.-S. Run, S.-J. Horng, J.-L. Lai, T.-W. Kao, and R.-J. Chen, "An improved SVD-based watermarking technique for copyright protection," Expert Systems with Applications, vol. 39, no. 1, pp. 673-689, 2012.

[34] M. Ali and C. W. Ahn, "Comments on 'optimized gray-scale image watermarking using DWT-SVD and Firefly Algorithm'," Expert Systems with Applications, vol. 42, no. 5, pp. 2392-2394, 2015.

[35] J.-M.Guo and H. Prasetyo, "Security analyses of the watermarking scheme based on redundant discrete wavelet transform and singular value decomposition," AEU-International Journal of Electronics

[36] C.-C. Lai, "An improved SVD-based watermarking scheme using human visual characteristics," Optics Communications, vol. 284, no. 4, pp. 938-944, 2011.

[37] Zheng, Y., Wu, C. H., Lu, Z. M., \& Ip, W. H. "Optimal robust image watermarking based on PSO and HVS in integer DCT domain", International Journal of Computer Sciences and Engineering System, Vol.2, No.4, pp.281-287, 2008.

[38] Vahedi, E., Lucas, C., Zoroofi, R. A., \& Shiva, M,“A new approach for image watermarking by using particle swarm optimization”,In Proceedings of IEEE ICSPC, 2007, pp. 1383-1386.

[39] R. Surya Prakasa Rao, Dr. P. Rajesh Kumar, "An Efficient Genetic Algorithm Based Gray scale Digital Image watermarking for Improving the Robustness and Imperceptibility", In Proc., of International Conference on Electrical, Electronics, and Optimization Techniques (ICEEOT), 2016.

[40] Hai Tao, Jasni Mohamad Zain, "A wavelet-based particle swarm optimization algorithm for digital image watermarking", Integrated Computer-Aided Engineering,VOl.19, pp.81-91, 2012.

[41] R. Surya Prakasa Rao, Dr. P. Rajesh Kumar, "PSO Based Lossless And Robust Image Watermarking Using Integer Wavelet Transform", GJCST, 2016. 